\title{
Synchrotron self-absorption in the GPS radio galaxy B0108+388
}

\author{
V. S. Artyukh ${ }^{1,2}$, P. A. Chernikov ${ }^{2}$, and S. A. Tyul'bashev ${ }^{1,2}$ \\ 1 Isaac Newton Institute of Chile, Pushchino Branch, Chile \\ e-mail: art@prao.ru \\ 2 Pushchino Radio Astronomy Observatory, P.N. Lebedev Physical Institute, Russian Academy of Sciences, Pushchino, \\ Moscow Region 142290, Russia
}

Received 21 December 2007 / Accepted 4 April 2008

\section{ABSTRACT}

\begin{abstract}
Context. The compact radio source in the nucleus of the GPS radio galaxy B0108+388 consists of two jets that have spectra that turn over at frequecies lower than $\sim 5 \mathrm{GHz}$. There is evidence that the spectral turnover in B0108+388 results from free-free absorption by non-uniform thermal plasma in central tens of parsecs.

Aims. To reveal the real cause of the low frequency cutoff in the jet spectra, it is necessary to consider different mechanisms and, in particular, syncrotron self-absorption.

Methods. We suggest that spectral turnovers at low frequencies of the jets are caused by synchrotron self-absorption. Estimations of physical parameters of these jets were obtained with a method based on a non-uniform synchrotron source model.

Results. We find that magnetic fields in these jets are nearly uniform with $10^{-2}<H_{\perp}<10^{-1} \mathrm{G}$ and with relativistic electron density $10^{1}<n_{\mathrm{e}}<10^{2} \mathrm{~cm}^{-3}$. The relativistic electron energy is higher everywhere in the jets than the magnetic field energy, but by taking the estimated uncertainties into account it is possible that energy equipartition is in effect. We compare the physical conditions in the jets and in the nuclei of classical doubles.

Conclusions. We present evidence that the spectral turnover in the B0108+388 results from synchrotron self-absorption.
\end{abstract}

Key words. galaxies: active - galaxies: magnetic fields - galaxies: jets - radio continuum: galaxies - methods: data analysis

\section{Introduction}

The radio source B0108+388 is characterized by a convex radio spectrum peaking at gigahertz frequencies. Such sources are called gigahertz peaked-spectrum (GPS) radio sources. GPS sources have steep spectral indices at high frequencies and spectral turnovers (low frequency cutoffs) near $1 \mathrm{GHz}$ (Gopal Krishna et al. 1983; Spoelstra et al. 1985; O’Dea et al. 1991). These radio sources are found mainly in distant highredshift galaxies and characterized by small angular dimensions $\sim 0.01^{\prime \prime}-0.1^{\prime \prime}$, which equals $\sim 10-1000 \mathrm{pc}$ on a linear scale (Mutel \& Phillips 1988).

GPS radio sources are characterized by a high radio luminosity of $\sim 10^{45} \mathrm{erg} / \mathrm{s}$, which is comparable to the luminosity of high-intensity quasars. However, their high luminosity is not consistent with Doppler-boosted jets and appears to be the true luminosity of these radio sources (Phillips \& Mutel 1982; Wilkinson et al. 1994). The GPS radio sources have low polarization and variability in luminosity (Rudnick \& Jones 1982). A detailed outline of radio, optical, and X-ray studies is given in the comprehensive review by O'Dea (1998).

Optical identification of GPS radio sources has revealed active galaxies of different types: quasars, BL Lac sources, and radio galaxies. We note that almost all GPS quasars have complicated asymmetric structure in VLBI maps. At the same time, practically all GPS radio galaxies are two-component radio sources with components similar in flux density and radio spectrum shape (Philips \& Mutel 1982; Wilkinson et al. 1994; Readhead et al. 1996).

At present three hypotheses about the nature of GPS sources are under consideration.
(1) GPS sources are old small-sized formations surrounded by dense environment preventing them from expanding to the size of typical nearby radio galaxies (van Breugel et al. 1984);

(2) GPS sources are very young formations (protogalaxies) that evolve through a compact steep spectrum (CSS) stage on their way to become large sources (Shklovsky 1965; Blake 1970; Readhead \& Hewish 1976; Phillips \& Mutel 1982; Carvalho 1985). Detected self-motion of components of some GPS sources made it possible to estimate their kinematic age of $\sim 10^{3}$ years (Taylor et al. 2000) proving that they are young objects;

(3) Tracking of extended components in a few sources gave rise to the third hypothesis that GPS sources are old objects characterized by recurrent activity of the galactic nucleus (Baum et al. 1990; Readhead et al. 1996). It seems possible that different GPS sources have different natures.

There is no unanimity as to the nature of the turnovers of GPS radio source spectra. It is accepted that at high-frequencies (exceeding the frequency of the spectrum peak) the power law spectrum of an object goes perfectly with the idea of synchrotron radiation of an electron ensemble with power law distribution of energies. As for low-frequency cutoffs, four mechanisms are discussed in the literature:

(1) synchrotron self-absorption (Slish 1963; Kellermann 1966; Jones et al. 1974);

(2) free-free absorbtion (Kellermann 1966; de Bruyn 1976);

(3) induced Compton scattering (Sunyaev 1970; Kuncic et al. 1998);

(4) the Razin effect (Razin 1960). 
Radio source B0108+388 was identified by Lawrence et al. (1996) with a weak galaxy $\left(m_{v}=22.0\right)$ with redshift $z=$ 0.67027 . Optical images show a very red, diffuse, and slightly asymmetric formation that is likely to be a face-on spiral galaxy (Stanghellini et al. 1993). Galaxy monitoring determined radiation variability in the near infrared region (Stickel et al. 1996).

The radio source center is located $0.5^{\prime \prime}$ from the optical image center. Its $4.8 \mathrm{GHz}$ radiation is slightly polarized $(0.3 \% \pm 0.08 \%)$, and no considerable radiation variability is evident (Aller et al. 1992). First VLBI observations at $5 \mathrm{GHz}$ revealed the simple dual structure of the radio source (Pearson \& Readhead 1988). Taylor et al. (1996) made more sensitive and higher resolution VLBA observations at higher frequencies (8.4 and $15 \mathrm{GHz}$ ) that revealed that the radio source on a millisecond scale consisting of seven components (a chain of components with S-symmetry). Outermost components move apart with an apparent velocity of $0.197 \pm 0.026 h^{-1} c$ according to Owsianik et al. (1998), and $0.24 \pm 0.04 h^{-1} c$ according to Taylor et al. (2000). The estimated kinematic age of the radio source is correspondingly $367 \pm 48$ years and $310 \pm 70$ years, making this the youngest radio galaxy among galaxies known to date. However, observations made at $6 \mathrm{~cm}$ and $49 \mathrm{~cm}$ with the WRST radio telescope and at $20 \mathrm{~cm}$ with VLA revealed the presence of an $\sim 3^{\prime \prime}$ extended radio source at the distance $\sim 20^{\prime \prime}$ to the east of the nucleus and connected to the nucleus with a faint bar (Baum et al. 1990). The presence of radio emission tens of $\mathrm{kpc}$ from the $\mathrm{B} 0108+388$ nucleus is inconsistent with any recent origin for the radio activity in this source. Baum et al. (1990) assume either that the activity in this object is recurrent and the extended emission is the relic of a previous epoch of activity or that this an old radio galaxy with its activity suppressed by dense gas within the galactic nucleus. The latter seems quite possible if the parent galaxy of B0108+388 radio source has emerged due to the merging of two galaxies rich in gas.

As mentioned above, VLBA observations of the B0108+388 radio source at 8.4 and $15 \mathrm{GHz}$ (Taylor et al. 1996) made it possible to identify 7 components. From now on, we will use the numbering of the source components introduced in this paper. The brightest are the outermost components: southwest (C1) and northeast (C7). A very compact (unresolved) component $\mathrm{C} 3$ with an exceptionally steep inverted spectrum $(\alpha \simeq-3 \pm 0.3)$ in $8.4-15 \mathrm{GHz}$ range was singled out. The authors think that this component is connected to the central engine emitting two jets in opposite directions. Subsequent papers confirmed the inverted spectrum of $\mathrm{C} 3$ component but the spectral index is $\alpha=-1.3 \pm 0.4$ in (Taylor et al. 2000), and $\alpha=-1.8 \pm 0.36$ (Marr et al. 2001) in the same frequency range of $8-15 \mathrm{GHz}$.

Marr et al. (2001) observed the radio source B0108+388 with the VLBA system at frequencies 15.359, 8.417, 4.983, 2.267, and $1.663 \mathrm{GHz}$ and obtained spectra of both jets. More specifically they obtained spectra of peak flux density values of the jets using the maps with the same resolution at all frequencies. The authors suggest that observed low-frequency cutoffs of jet flux density peaks could occur due to free-free absorption of the synchrotron radiation by thermal plasma inside a disk of ionized gas between the radio source and the observer. Lack of noticeable polarization at $5 \mathrm{GHz}$ testifies that high-density thermal plasma can exist in the galaxy (Cawthorne et al. 1993). A high content of neutral hydrogen in B0108+388 detected in absorption line $21 \mathrm{~cm}$ (Carilli et al. 1998) also supports the suggested interpretation. As said above, the low frequency cutoff of the jet spectra can also be caused by in principle, induced Compton scattering or by the Razin effect, but we are unaware of any work that has applied these mechanisms to interpreting the jet spectra in $\mathrm{B} 0108+388$.

We report here that the spectrum of the compact radio source B0108+338 can be described in full by the synchrotron mechanism of radiation and absorption. Under the hypothesis of the synchrotron mechanism of a radiation radio source physical parameters were estimated using the method of Artyukh \& Chernikov (2006).

\section{The method of physical parameter estimations}

To estimate physical parameters of radio sources, we use the method developed in Artyukh \& Chernikov (2006). This method is based on a non-uniform synchrotron source model. Distributions of the magnetic field strength and the relativistic electron density are approximated by the functions:

$$
\begin{array}{ll}
H(r)=\frac{H(0)}{1+k_{\mathrm{H}}\left(\frac{r}{R}\right)^{m}}, & \text { if } \quad r \leq R, \quad \text { and } \\
H(r)=0, \quad \text { if } \quad r>R, & \\
N(E, r)=E^{-\gamma} \frac{N(0)}{1+k_{N}\left(\frac{r}{R}\right)^{n}}, & \text { if } r \leq R, \text { and } \\
N(E, r)=0, & \text { if } \quad r>R,
\end{array}
$$

where $R$ is source model radius. Thus, a radio source model is characterized by the following physical parameters: $\gamma$ (power index of the energy spectrum $N(E) \sim E^{-\gamma}$ ), $R, H(0)$ (magnetic field strength in the center of the source), $m$ (power index of the magnetic field distribution), $k_{H}$ (coefficient of the magnetic field contrast), $N(0)$ (relativistic electron density in the center of the source), $n$ (power index of the particle distribution), $k_{N}$ (coefficient of the particle distribution contrast).

According to Condon \& Dressel (1973); de Bruyn (1976); \& Artyukh \& Chernikov (2001) the spectrum of such a synchrotron source consists of three parts. At high frequencies the flux density is $S \sim v^{-\alpha}$ in transparent wave range $\left(v>v_{\mathrm{hf}}\right)$, where $v_{\mathrm{hf}}$ is the frequency of the high-frequency break in the spectrum. At low frequencies $S$ is $\sim v^{2.5}$ in the opacity region $\left(v<v_{\mathrm{lf}}\right)$, where $v_{\mathrm{lf}}$ is the frequency of the low-frequency break in the spectrum. In many cases the spectrum at intermediate frequencies $\left(v_{\mathrm{lf}}<v<v_{\mathrm{hf}}\right)$ can be described by a power function, too: $S \sim v^{-\alpha_{\text {lf }}}$, where $-2.5<\alpha_{\text {lf }}<\alpha$.

All physical parameters of a compact radio source are determined from radio astronomy observations. To obtain the physical information we must have the spectrum of the radio source with the high frequency cutoff (at a frequency $v_{\mathrm{hf}}$ ) and the low frequency cutoff (at a frequency $v_{\text {lf }}$ ), the high frequency spectral index $\alpha$, the low frequency spectral index $\alpha_{\mathrm{lf}}$, angular diameters of the radio source at different frequencies, and the redshift of the host galaxy.

Source physical parameters are calculated as follows. From observations at higher frequencies, where a source is transparent and $S \sim v^{-\alpha}$, we have $\gamma=2 \alpha+1$. The parameter $k_{H}$ is determined by the frequency interval length $v_{\mathrm{hf}}-v_{\mathrm{lf}}$. Thus it can be obtained from the model catalog (Artyukh \& Chernikov 2001) if $v_{\mathrm{hf}}-v_{\mathrm{lf}}$ is known from observations. We have from observations the spectral index $\alpha_{\mathrm{lf}}$ (within $v_{\mathrm{lf}}<v<v_{\mathrm{hf}}$ range). From de Bruyn (1976)

$$
\alpha_{\mathrm{lf}}=\frac{13-5 n-3 m-2 m \gamma+2 \gamma}{2-2 n-2 m-m \gamma}
$$

If $\alpha_{\text {lf }}$ and $\gamma$ are known, a linear relation between $m$ and $n$ can be derived from Eq. (3). As shown in Artyukh \& Chernikov (2001), parameters $m$ and $n$ belong to the finite interval of values that 
corresponds to physically realizable source models. To simplify the work we choose the model with a uniform distribution of particles, i.e. $n=0, k_{N}=0$, and $N(E)=N_{0} E^{-\gamma}$. Thus we derive $\mathrm{m}$ directly from (3). As shown in Artyukh \& Chernikov (2001) in this case we deal with the source model with minimum magnetic field $H(0)$ and maximum particle density $N_{0}$. It should be noted that another marginal model with $m=0$ offers maximum magnetic field that depends on the model itself. The maximum magnetic field in models close to uniform is an order of magnitude greater than the minimum field, and in highly non-uniform models - almost by two orders. The minimum density of particles is an order of magnitude lower than the maximum density. Thus it follows that we have actually got an interval estimation of the parameters by obtaining the minimum estimation of magnetic field strength $H(0)$ and the maximum estimation $N_{0}$.

To estimate parameters $N_{0}, R$, and $H(0)$, we use the following set of equations:

$$
\begin{aligned}
\tau\left(v_{\mathrm{hf}}\right) & =\int_{-R}^{R} c_{6}(\gamma) N_{0} H^{\frac{\gamma+2}{2}}(x)\left({\frac{v_{\mathrm{hf}}}{2 C_{1}}}^{\frac{-\gamma-4}{2}}\right) \mathrm{d} x \simeq 1, \\
\frac{I\left(v_{\theta}, r_{\theta}\right)}{I\left(v_{\theta}, 0\right)} & =\frac{1}{2} \\
S\left(v_{i}\right) & =\int_{\Omega} I\left(v_{i}, \omega\right) \cos (\theta) \mathrm{d} \omega \simeq \int_{\Omega} I\left(v_{i}, \omega\right) \mathrm{d} \omega,
\end{aligned}
$$

where $\Omega$ is the solid angle of a radio source.

The first equation requires that the optical depth along the model diameter at the high-frequency break, $v_{\mathrm{hf}}$, be equal to one (Artyukh \& Chernikov 2001). The second equation requires the angular diameter of the source model at the observation frequency $v_{\theta}$ to be equal to the measured angular diameter of the real radio source at the same frequency $\left(r_{\theta}-\right.$ half-power model radius). The third equation requires that the flux density of source model radiation equals the measured flux density $S\left(v_{i}\right)$ of the source.

Intensity $I(v, r)$ is obtained from the solution of the transfer equation (Mustel 1960). For synchrotron emission we have

$$
\begin{aligned}
& I(v, r)=\int_{-L}^{L} c_{5}(\gamma) N_{0} H^{\frac{\gamma+1}{2}}(x) \\
& \times\left(\frac{v}{2 C_{1}}\right)^{\frac{1-\gamma}{2}} \mathrm{e}^{-\int_{x}^{L} c_{6}(\gamma) N_{0} H^{\frac{\gamma+2}{2}}\left(x^{\prime}\right)\left(\frac{\gamma}{2 C_{1}}\right)^{\frac{-4-\gamma}{2}} \mathrm{~d} x^{\prime}} \mathrm{d} x
\end{aligned}
$$

where $L=\left(R^{2}-r^{2}\right)^{1 / 2}$. The values of $c_{5}(\gamma), c_{6}(\gamma)$ functions and $C_{1}$ factor are given in Pacholczyk (1970). Using estimated model parameters, we calculate its spectrum from Eq. (7).

Thus all physical parameters of the radio source model are estimated and its spectrum calculated. It is necessary to emphasize that, because of the paucity of observation data, it is possible to obtain only interval estimations of the physical parameters.

As shown in Artyukh \& Chernikov (2006), the basic error of physical parameter estimation relates to the estimation error of the radio source angular dimension, and the $H(0)$ and $N(0)$ estimation error can come up to the order of magnitude. This is appropriate only when one-frequency angular dimension of the radio source is obtained from observations. If angular dimensions are available for two (or more) frequencies in the transparent wave range and in the partially opacity wave range, the accuracy of the estimation is increased considerably.

\section{Results of the investigations}

According to Taylor et al. (1996), we assume that the compact radio source in $\mathrm{B} 0108+388$ radio galaxy observed on a milliarcsecond scale comprises two jets emitted by the central engine
Table 1. Flux density values for the jets of B0108+388 radio galaxy.

\begin{tabular}{cccc}
\hline \hline$v(\mathrm{GHz})$ & $S_{N-E}(\mathrm{Jy})$ & $S_{S-W} \mathrm{Jy}$ & References \\
\hline 90 & $<0.16$ & $<0.16$ & 1 \\
15 & 0.428 & 0.15 & 2 \\
15 & 0.334 & 0.14 & 3 \\
8.5 & 0.59 & 0.28 & 4 \\
5 & 1.16 & 0.56 & 5 \\
5 & 0.86 & 0.51 & 6 \\
2.3 & 0.71 & 0.46 & 4 \\
1.67 & 0.27 & 0.30 & 7 \\
0.6 & $<0.075$ & $<0.075$ & 8 \\
\hline
\end{tabular}

(1) Steppe et al. (1995); (2) Taylor et al. (1996); (3) Taylor et al. (2000); (4) Fey \& Charlot (1997); (5) Pearson \& Readhead (1988); (6) Fomalont et al. (2000); (7) Polatidis et al. (1995); (8) Stanghellini et al. (1998). Integral flux densities at frequencies 0.6 and $90 \mathrm{GHz}$ are the upper limits for each jet.
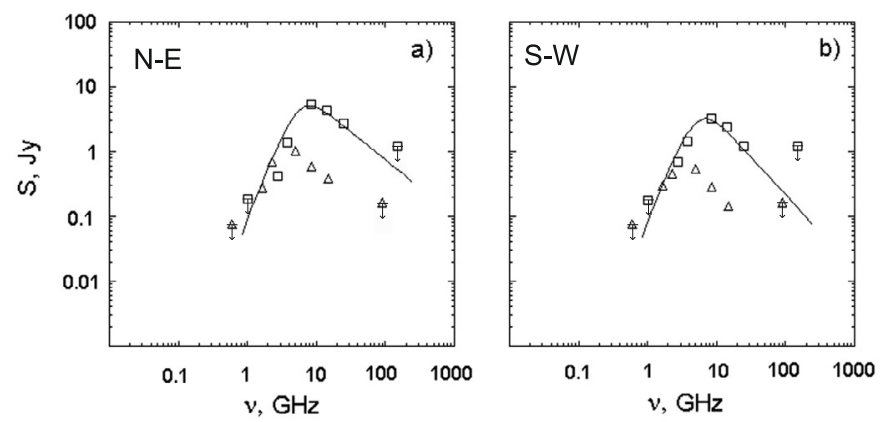

Fig. 1. Spectra of B0108+388 jets: a) the spectrum of the northeast jet, b) the spectrum of the southwest jet. Observed spectra are shown by triangles, corrected spectra - by squares. Calculated model spectra are shown by solid lines.

identified with the $\mathrm{C} 3$ component. It is assumed that each jet is an independent physical system. Flux densities of the jet components were taken from the literature. High-frequency flux densities of each jet were obtained by summing up flux densities of separate components: components $\mathrm{C} 1$ and $\mathrm{C} 2$ for the southwest jet and components C4, C5, C6 and C7 for the northeast jet. At low-frequencies the resolution capability of earth-based interferometers was insufficient to single out jets' separate components. In those cases we made use of the jet's flux density values given by the authors of measurements. Jet flux density values are given in Table 1. Unfortunately we have no information about the errors of the flux densities, so when fitting the calculated spectrum from Eq. (7) to the measured flux densities we have assumed an error of $\sim 15 \%$ on all flux densities.

The parameter estimation method outlined above was applied to the radio source in its observed frame. The B0108+388 radio galaxy is moving away from the Earth $(z=0.67)$. Thus, to estimate jets' physical parameters we must reconstruct their spectra for the source frame. All frequencies were increased by a factor $(1+z)$. Flux densities were increased by a factor $(1+z)^{3+\alpha}$, considering that $\alpha$ varies with the frequency. Spectra of the jets are shown in Fig. 1a (for the northeast jet) and 1b (for the southwest jet). The spectra, obtained from the numerical solution of the transfer equation, are shown by solid lines.

The jets' angular dimensions were taken from VLBI observations at $15 \mathrm{GHz}$ (Taylor et al. 2000). The linear angular dimension $(\theta)$ of each jet model equals the mean geometric value of solid angles $(\Omega)$ for all components. The measured angular dimension $\theta$ was reduced by a factor of $(1+z)$ with allowance 

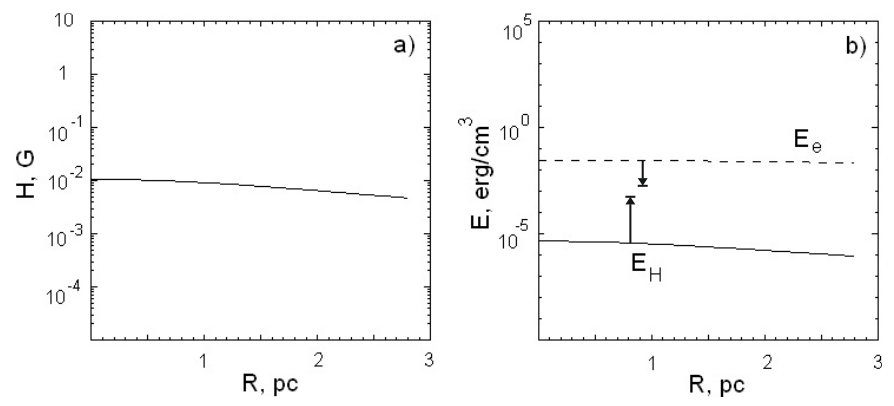

Fig. 2. Physical parameters of the northeast model jet: a) the distribution of the minimum magnetic field strength, $\mathbf{b}$ ) the distribution of minimum energy of the magnetic field and maximum energy of the relativistic electrons.
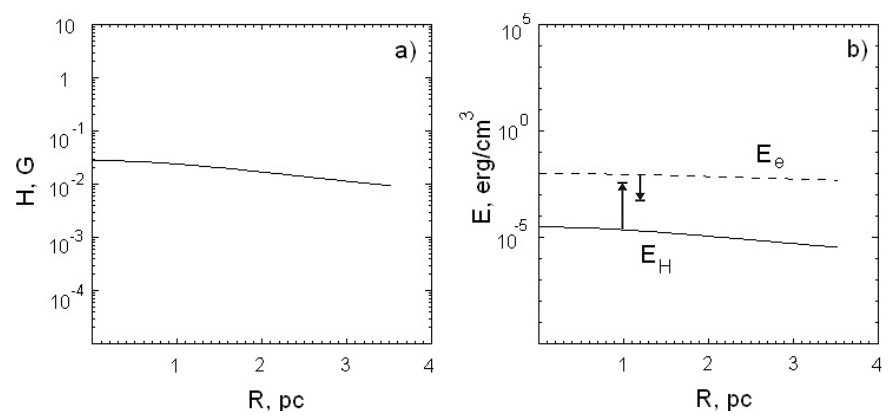

Fig. 3. Physical parameters of the southwest model jet: a) the distribution of the minimum magnetic field strength, b) the distribution of minimum energy of the magnetuc field and maximum energy of the relativistic electrons.

for aberration. The calculations of the jet's linear dimensions were made using the NED Cosmology Calculator I program for the planar model of the universe with the following parameters $H_{0}=71 \mathrm{~km} \mathrm{~s}^{-1} / \mathrm{Mpc}, \Omega_{\mathrm{M}}=0.27, \Omega_{\mathrm{vac}}=0.73$.

The analysis results are given in Figs. 2 and 3. Figure $2 \mathrm{a}$ shows the distribution of the magnetic field strength $(\mathrm{H})$ in the northeast jet model within the distance to the center (r). The figure presents the minimum magnetic field. This magnetic field corresponds to the maximum density of relativistic electrons $\left(N_{0}\right)$. Figure $2 \mathrm{~b}$ shows the distribution of minimum energy of the magnetic field and maximum energy of the relativistic electrons. Similar values are given for the southwest jet in Figs. 3a, b.

As seen in Fig. 2a and 3a, the distributions of magnetic fields in jets are very close to a uniform distribution. The results of physical parameters estimation of both jets are similar. $H(0)=$ $1.0 \times 10^{-2} \mathrm{G},\langle H\rangle=6 \times 10^{-3} \mathrm{G}, n_{\mathrm{e}}=370 \mathrm{~cm}^{-3}$ for the northeast jet on a 3 pc scale. $H(0)=2.7 \times 10^{-2} \mathrm{G},\langle H\rangle=1.3 \times 10^{-2} \mathrm{G}$, $n_{\mathrm{e}}=180 \mathrm{~cm}^{-3}$ for the southwest jet on a $4 \mathrm{pc}$ scale. These are the lower values for the magnetic field strength and the upper values for particle densities, i.e. maximum $H(0)$ value can be an order of magnitude greater since the field distribution is close to uniform and minimum density of relativistic electrons can be an order of magnitude smaller (Artyukh \& Chernikov 2006).

Calculated spectra of synchrotron source models with the above parameters are shown in Figs. 1a,b. As seen in the figure, the jet spectra can be described by synchrotron source models without involving radio radiation absorption by a thermal plasma screen. Thus, magnetic fields inside the jets are distributed almost uniformly. The magnetic field strength lies within the range $10^{-2}<H<10^{-1} \mathrm{G}$, and the relativistic electrons density lies within the range $10^{2}>n_{\mathrm{e}}>10^{1} \mathrm{~cm}^{-3}$ (at $\sim 10 \mathrm{pc}$ scale). Everywhere in the jets the relativistic electron energy is higher than the magnetic field energy, but if taking the estimated uncertainties into account it is possible energy equipartition is in effect.

Observations of the entire spectrum of the central component C3 are unfortunately not available, so we cannot apply our method to estimate the physical parameters. However, as stated above, observations at 8 and $15 \mathrm{GHz}$ revealed an inverted spectrum for this component within the range $8-15 \mathrm{GHz}$. If the lowfrequency cutoff of C3's spectrum at such high frequencies is caused by synchrotron self-absorption, it could be indicative of a strong magnetic field strength with the upper limit $\sim 7 \times 10^{4} \mathrm{G}$ (Taylor et al. 2000). Since C3 component is unresolved, this would imply that the strong magnetic field must exist on a scale $<1$ pc.

\section{Discussion}

It has been suggested that GPS sources evolve into CSS sources and eventually into classical double radio galaxies (Phillips \& Mutel 1982; Mutel \& Phillips 1988). Carvalho (1985) presented a simple analytical model of the evolution of classical double sources that is consistent with such a scenario. It is interesting to compare physical conditions in young jets of the GPS radio galaxy B0108+388 and in nuclei of classical doubles.

A study of physical conditions in the nuclei of nearby (old) radio galaxies $(z<0.05) 3 \mathrm{C} 111$ and $3 \mathrm{C} 465$ was carried out by Chernikov et al. (2006) using the same method as applied here to $\mathrm{B} 0108+388$. A strong non-uniform magnetic field distribution in the nuclei of these radio galaxies was found. The magnetic field strength in the center of nuclei 3C 111 and 3C 465 is $10^{2}<H<10^{4} \mathrm{G}$ at $\sim 0.1 \mathrm{pc}$ scale for the average magnetic field $\langle H\rangle \sim 10^{-2} \mathrm{G}$ (on the scale $\sim 10 \mathrm{pc}$ ) in $3 \mathrm{C} 111$ and $\langle H\rangle \sim 10^{-1} \mathrm{G}$ (on the scale $4 \mathrm{pc}$ ) in $3 \mathrm{C} 465$. The relativistic electron density is $10^{-5}<n_{\mathrm{e}}<10^{-4} \mathrm{~cm}^{-3}$. No energy equipartition was found in the nuclei of the radio galaxies. Everywhere in the nuclei the relativistic electron energy is lower than the magnetic field energy in opposite to B0108+388. Thus, magnetic fields in B0108+388 jets are similar to magnetic fields in nuclei of 3C 111 and 3C 465 (at the same scale), but relativistic electron densities in young jets of B0108+388 are much more than in cores of 3C 111 and $3 \mathrm{C} 465$. These differences in physical conditions most likely are connected to an evolution of classical doubles.

It follows from our analysis that jet magnetic fields are distributed almost uniformly, hence jet boundaries demonstrate abrupt magnetic field jumps. This is in accord with the shock wave concept, and it may be that the jets are propagating in the interstellar medium like shock waves.

We have shown that the spectral turnover observed in B0108+388 is consistent with synchrotron self-absorption. However Marr et al. (2001) come to the conclusion that free-free absorption is responsible. As yet the available radio astronomy data do not exclude either of these two spectral turnover mechanisms. However at frequencies $<0.6 \mathrm{GHz}$, the spectra will be different for each of these mechanisms, making it necessary to perform VLBI observations at very low frequencies $(<0.6 \mathrm{GHz})$ to obtain the low frequency spectrum of the source in detail and to reach a final conclusion.

\section{Conclusion}

We have proposed that the low frequency cutoffs in spectra of the jets in the GPS radio galaxy B0108+388 are conditioned by synchrotron self-absorption. It was found that magnetic fields in 
these jets are nearly uniform with $10^{-2}<H_{\perp}<10^{-1} \mathrm{G}$ and with relativistic electron density $10^{-1}<n_{e}<10^{2} \mathrm{~cm}^{-3}$. Everywhere in the jets, the relativistic electron energy is higher than the magnetic field energy, but when taking the estimated uncertainties into account it is possible that energy equipartition is in effect.

The physical parameters of the jets in $\mathrm{B} 0108+388$ were compared with physical parameters of the nuclei of nearby radio galaxies 3C 111 and 3C 465. These parameters were obtained by the same method and at the same scale. It was found that the magnetic fields are similar but the relativistic electron density in the jets of $\mathrm{B} 0108+388$ is much higher than found in the cores of 3C 111 and 3C 465. Everywhere in the nuclei of 3C 111 and $3 \mathrm{C} 465$, the relativistic electron energy is lower than the magnetic field energy, in contrast to what is found in B0108+388. This implies that the physical conditions in the nuclei of radio galaxies may change during the course of their evolution, if GPS radio galaxies represent the early stage of the evolution of classical double radio galaxies.

We present evidence that the radio spectral turnover in the jets of B0108+388 results from synchrotron self-absorption rather than the free-free absorption favored by Maar et al.(2001). VLBI observations of the radio source at frequencies lower than $0.6 \mathrm{GHz}$ are needed to separate these two mechanisms.

Acknowledgements. This work was supported by the Russian Foundation for Basic Research (project code 05-02-17011). This research made use the NASA Extragalactic Database (NED). We thank the anonymous referee for the helpful suggestions and comments.

\section{References}

Aller, M. F., Aller, H. D., \& Hughes, P. A. 1992, ApJ, 399, 16 Artyukh, V. S., \& Chernikov, P. A. 2001, Astron. Rep., 45, 16

Artyukh, V. S., \& Chernikov, P. A. 2006, Astron. Rep., 50, 194

Blake, G. M. 1970, ApJ, 6, L201

Baum, S. A., O’Dea, C. P., Murphy, D. W., \& de Bruyn, A. G. 1990, A\&A, 232, 19

Carilli, C. L., Menten, K. M., Reid, M. J., Rupen, M. P., \& Yun, M. S. 1998, ApJ, 494, 175

Carvalho, J. C. 1985, MNRAS, 215, 463
Cawthorne, T. V., Wardle, J. F. C., Roberts, D. H., Gabuzda, D. C., \& Brown, L. F. 1993, ApJ, 416, 496

Chernikov, P. A., Artyukh, V. S., Tyul'bashev, S. A., \& Lapaev, K. A. 2006, Astron. Rep., 50, 202

Condon, J. J., \& Dressel, L. L. 1973, ApJ, 15, L203

de Bruyn, A. G. 1976, A\&A, 52, 439

Fey, A. L., \& Charlot, P. 1997, ApJS, 111, 95

Fomalont, E. B., Frey, S., Paragi, Z., et al. 2000, ApJS, 131, 95

Gopal-Krishna, Patnaik, A. R., \& Steppe, H. 1983, A\&A, 123, 107

Jones, T. W., O’Dell, S. L., \& Stein, W. A. 1974, ApJ, 192, 261

Kellermann, K. I. 1966, Australian. J. Phys., 19, 195

Kuncic, Z., Bicknell, G. V., \& Dopita, M. A. 1998, ApJ, 495, L35

Lawrence, C. R., Zucker, J. R., Redhead, A. C. S., Unwin, S. C., Pearson, T. J., \& Xu, W. 1996, ApJS, 107, 541

Marr, J. M., Taylor, G. B., \& Crawford, F. 2001, ApJ, 550, 160

Mustel, E. R. 1960, Stellar atmospheres, Fiz-Mat. Literaturi, Moscow

Mutel, R. L., \& Phillips, R. B. 1988, The Impact of VLBI on Astrophysics and Geophysics, ed. Reid, M. J., Moran, J. M. (Dordrecht: Kluwer), IAU Symp. 129,73

O’Dea, C. P. 1998, PASP, 110, 493

O'Dea, C. P., Baum, S. A., \& Stanghellini, C. 1991, ApJ, 380, 66

Owsianik, I., Conway, J. E., \& Polatidis, A. G. 1998, A\&A, 336, L37

Pacholczyk, A. G. 1970, Radio Astrophysics, W. H. (San Francisco: Freeman and company)

Pearson, T. J., \& Readhead, A. C. S. 1988, ApJ, 328, 114

Phillips, R. B., \& Mutel, R. L. 1982, A\&A, 106, 21

Polatidis, A. G., Wilkinson, P. N., Xu, W., et al. 1995, ApJS, 98, 1

Razin, V. A. 1960, Izv. Vyssh. Uchebn. Zaved. Radiofizika, 3, 921

Readhead, A. C. S., \& Hewish, A. 1976, MNRAS, 176, 571

Readhead, A. C. S., Taylor, G. B., Xu, W., et al. 1996, ApJ, 432, L87

Rudnick, L., \& Jones, T. W. 1982, ApJ, 255, 39

Shklovsky, I. S. 1965, Nature, 206, 176

Slish, V. I. 1963, Nature, 199, 682

Spoelstra, T. A. T., Patnaik, A. R., \& Gopal-Krishna 1985, A\&A, 152, 38

Stanghellini, C., O'Dea, C. P., Baum, S. A., \& Laurikanen, E. 1993, ApJS, 88, 1 Stanghellini, C., O’Dea, C. P., Dallacasa, D., et al. 1998, A\&AS, 131, 303

Steppe, H., Jeyakumar, S., Saikia, D. J., \& Salter, C. J. 1995, A\&AS, 113, 409

Stickel, M., Rieke, G. H., Kuhr, H., \& Rieke, M. J. 1996, ApJ, 468, 556

Sunyaev, R. A. 1970, ApJ, 7, L19

Taylor, G. B., Readhead, A. C. S., \& Pearson, T. J. 1996, ApJ, 463, 95

Taylor, G. B., Marr, J. M., Pearson, T. J., \& Redhead, A. C. S. 2000, ApJ, 541, 112

Van Breugel, W., Miley, G., \& Heckman, T. 1984, AJ, 89, 5

Wilkinson, P. N., Polatidis, A. G., Readhead, A. C. S., Xu, W., \& Pearson, T. J. 1994, ApJ, 432, L87 